\title{
A sensitive and specific indirect competitive enzyme-linked immunosorbent assay for the detection of icariin
}

\author{
JINJUN CHENG ${ }^{1}$, YUE ZHANG ${ }^{2}$, SHUCHEN LIU ${ }^{1}$, BAOPING QU ${ }^{1}$, MEILING ZHANG ${ }^{3}$, \\ XIAOMAN LIU ${ }^{1}$, YAN ZHAO ${ }^{1}$ and HUIHUA QU ${ }^{4}$ \\ ${ }^{1}$ Department of Basic Traditional Chinese Medicine Clinical Science, School of Basic Medical Sciences; \\ Departments of ${ }^{2}$ Pharmaceutics of Traditional Chinese Medicine and ${ }^{3}$ Chemistry of Traditional Chinese Medicine, \\ School of Chinese Materia Medica; ${ }^{4}$ Department of Molecular Biology, Beijing Institute of Traditional Chinese Medicine, \\ Beijing University of Chinese Medicine, Beijing 100029, P.R. China
}

Received September 24, 2015; Accepted October 11, 2016

DOI: $10.3892 / \mathrm{mmr} .2016 .5975$

\begin{abstract}
In the present study, a novel monoclonal antibody (MAb) specific for icariin (ICA) was prepared and characterized. A hybridoma-secreting MAb against icariin was produced by fusing splenocytes immunized with an ICA-bovine serum albumin conjugate with a hypoxanthine-aminopterin-thymidine-sensitive mouse myeloma SP2/0 cell line. The antibody showed high specificity for ICA with almost no cross-reactivity against the majority of structurally-related chemicals. Subsequently, an indirect competitive enzyme-linked immunosorbent assay (ELISA) for ICA was established and characterized. In this assay, an effective measuring range of $10-1,000 \mathrm{ng} / \mathrm{ml}$ of ICA $\left(\mathrm{R}^{2}=0.9828\right)$ was detected. Intra- and inter-assay repeatability and precision were achieved with a relative standard deviation (RSD) of $<10 \%$. A mean recovery of $95-115 \%$ was obtained, with an RSD of $<10 \%$. In addition, the levels of ICA in traditional Chinese herbal prescriptions
\end{abstract}

Correspondence to: Dr Yan Zhao, Department of Basic Traditional Chinese Medicine Clinical Science, School of Basic Medical Sciences, Beijing University of Chinese Medicine, 11 Beisanhuandong Road, Chaoyang, Beijing 100029, P.R. China

E-mail: zhaoyandr@gmail.com

Dr Huihua Qu, Department of Molecular Biology, Beijing Institute of Traditional Chinese Medicine, Beijing University of Chinese Medicine, 11 Beisanhuandong Road, Chaoyang, Beijing 100029, P.R. China

E-mail: quhuihua@gmail.com

Abbreviations: BSA, bovine serum albumin; CBS, carbonate buffer solution; GPBS, $10 \mathrm{mg} / \mathrm{ml}$ gelatine in PBS; HAT, hypoxanthine-aminopterin-thymidine; HT, hypoxanthine-thymidine; ICA, icariin; MAb, monoclonal antibody; OVA, ovalbumin; PBS, phosphate-buffered saline; PBST, PBS with $0.05 \%$ Tween-20; PEG, polyethylene glycol; TCM, traditional Chinese medicine

Key words: icariin, monoclonal antibody, enzyme-linked immunosorbent assay, traditional Chinese medicine were determined, and correlation between the ELISA and high-performance liquid chromatography analyses of total ICA was obtained. These results demonstrated that a reliable ELISA method had been successfully developed to determine ICA in traditional Chinese herbs and may contribute to further clinical investigations.

\section{Introduction}

Folium Epimedii, also known as Yin Yang Huo in China, is derived from the genus Epimedium, which includes $E$. sagittatum Maxim., E. pubescens Maxim., E. koreanum Nakai and E.wushanense T.S. Ying. All these species have been used for hundreds of years to combat several diseases, including erectile dysfunction, fatigue, kidney disorders and joint pain (1). Folium Epimedii has been confirmed to be effective in the treatment of cardiovascular diseases (2), osteoporosis (3) and tumors (4).

Icariin (ICA), has been suggested to be an indicative constituent of Folium Epimedii. Previous studies have indicated that ICA exhibits positive effects in suppressing inflammation, and promoting cardiovascular functions $(5,6)$ and antitumor activities $(7,8)$. Furthermore, previous studies have shown that ICA suppresses cartilage and bone degradation in mice with collagen-induced arthritis (9), and inhibits cell growth and induces apoptosis in Burkett lymphoma cell lines (10).

Previously, the quantitative and qualitative analyses of ICA had been performed primarily using high-performance liquid chromatography (HPLC) and thin-layer chromatography (TLC) $(11,12)$. However, these methods have various limitations, including high cost, component degradation, prolonged duration, low recovery rates and complicated pretreatment, particularly for in vivo investigations of metabolism. Therefore, it is necessary to establish a novel, simple method for ICA analysis.

The enzyme-linked immunosorbent assay (ELISA) method, based on specific monoclonal antibodies (MAbs) has become an important methodology for the qualitative or quantitative analysis of food or natural products $(13,14)$. This method is rapid and requires only minimal sample pre-treatment. In addition, 
the method can be used simultaneously for a large number of samples. Previously, MAbs against certain compounds in traditional Chinese medicines (TCMs) have been reported (15-21); however, no MAbs specific for ICA have been described.

In our previous studies, preparations of MAbs have been developed against baicalin (15), puerarin (16), geniposide (17), glycyrrhizic acid (18), paeoniflorin (19), ginsensoide Re (20) and ginsenoside Rh1 (21), and their ELISA methods have been established. Consequently, these assays were applied to examine the pharmacokinetics and pharmacokinetic interactions between these bioactive compounds. The comparably low quantities of sample required, for example $5 \mu \mathrm{l}$ of serum, for the determination in mice is particularly beneficial for pharmacokinetic investigations. Furthermore, the development of immunoaffinity chromatography based on anti-gisenoside Rh1 MAb has been indicated as a potential method for the separation of epimers (21).

In the present study, the formation and characterization of an anti-ICA MAb were investigated, and an indirect competitive (ic)ELISA method was established. The MAb and icELISA were used to detect ICA in the complex chemical constituents of TCM. This icELISA method may be of use for further investigations of ICA.

\section{Materials and methods}

Chemicals and reagents. ICA was purchased from Welch Materials, Inc. (Shanghai, China; purity of 95\%). Sodium periodate was obtained from Sinopharm Chemical Reagent Co., Ltd. (Beijing, China). Bovine serum albumin (BSA), ovalbumin (OVA) and Freund's complete and incomplete reagents were obtained from Sigma-Aldrich; Merck Millipore (Darmstadt, Germany). All other chemicals and reagents were of analytical grade and were purchased from Sinopharm Chemical Reagent Co., Ltd.

The composition of the four TCM detected were as

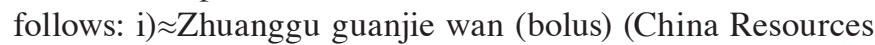
Sanjiu Medical and Pharmaceutical, Shenzhen, China): Cibotii Rhizoma, Epimedii Folium, Dipsaci Radix, Psoraleae Fructus, Spatholobi Caulis, Olibanum, Myrrha, Aucklandiae Radix, Drynariae Rhizoma, Taxilli Herba, Angelicae Pubescentis Radix, Rehmanniae Radix Praeparata; ii) Pishen Shuangbu decoction: Taxilli Herba (30 g), Corn Stigma (30 g), Polygoni Multiflori Radix (24g), Chuanxiong Rhizoma (9 g), Eucommiae Cortex (9 g), Magnetitum (3 g), Fossil Fragments (30 g) and Epimedii Folium (9 g); iii) Erxian decoction: Curculiginis Rhizoma (9 g), Epimedii Folium (9 g), Morindae officinalis Radix (9 g), Phellodendri Chinensis Cortex (4.5 g), Angelicae Sinensis Radix (9 g) and Anemarrhenae Rhizoma (4.5 g); iv) Tongbi Prescription: Aconiti Kusnezoffii Radix (15 g), Aconiti Radix (10 g), Asari Radix Et Rhizoma (6 g), Ephedrae Herba (10 g), Taxilli Herba (15 g), Zingiberis Rhizoma (10 g), Glycyrrhizae Radix Et Rhizoma (6 g), Epimedii Folium (15 g), Eucommiae Cortex (10 g) and Dipsaci Radix (15 g). All aforementioned Chinese herbal medicine was purchased from Tongrentang (Beijing, China).

The compositions of the buffers and solutions used in the present study were as follows: Phosphate-buffered saline (PBS; pH 7.4): $\mathrm{NaCl}(137 \mathrm{mmol} / \mathrm{l}), \mathrm{Na}_{2} \mathrm{HPO}_{4} \bullet 12 \mathrm{H}_{2} \mathrm{O}$ (10 mmol/l), $\mathrm{KCl}(2.68 \mathrm{mmol} / \mathrm{l})$ and $\mathrm{KH}_{2} \mathrm{PO}_{4}(1.47 \mathrm{mmol} / \mathrm{l})$; carbonate buffer solution (CBS; $\mathrm{pH} 9.6)$ : $\mathrm{Na}_{2} \mathrm{CO}_{3}(15 \mathrm{mmol} / \mathrm{l})$ and $\mathrm{NaHCO}_{3}$ (35 mmol/l); washing buffer: PBS with $0.05 \%$ Tween-20 (PBST); blocking buffer: $10 \mathrm{mg} / \mathrm{ml}$ gelatine in PBS (GPBS); tetramethylbenzidine (TMB) substrate solution: Combination of part A $(0.5 \mathrm{ml}$ of stock comprising $24.3 \mathrm{ml}$ of $0.1 \mathrm{~mol} / \mathrm{ml}$ citric acid, $25.7 \mathrm{ml}$ of $0.2 \mathrm{~mol} / \mathrm{ml} \mathrm{Na} \mathrm{HPO}_{4}$ and $50 \mathrm{ml}$ deionized water), part B (10 $\mathrm{ml}$ of stock comprising $2 \mathrm{mg}$ of TMB dissolved in $1 \mathrm{ml}$ of methanol) and part $\mathrm{C} \mathrm{(32 \mu \textrm {l }}$ of $0.75 \% \mathrm{H}_{2} \mathrm{O}_{2}$ ); stopping solution: $2 \mathrm{~mol} / \mathrm{ml} \mathrm{H}_{2} \mathrm{SO}_{4}$ and $1 \mathrm{mM}$ $\mathrm{HCl}(\mathrm{pH} 4.0 ; 20 \mathrm{ml} / \mathrm{g}$ ); hypoxanthine-aminopterin-thymidine (HAT); polyethylene glycol (PEG); and hypoxanthine-thymidine (HT).

Synthesis of ICA antigen conjugate. The conjugates were synthesized using a periodate oxidation procedure according to a previously reported protocol with modifications $(22,23)$. Briefly, the ICA was dissolved in CBS at $1 \mathrm{mg} / \mathrm{ml}$, following which $1 \mathrm{ml}$ of freshly prepared sodium periodate solution (8 $\mathrm{mg}$ ) was added drop-wise to $5 \mathrm{ml}$ of the ICA solution. The mixture was stirred at $25^{\circ} \mathrm{C}$ for $1 \mathrm{~h}$, following which $5 \mathrm{mg}$ of BSA dissolved in $1 \mathrm{ml}$ of $\mathrm{CBS}$ was added, and the final $\mathrm{pH}$ was adjusted to 9.0 using $0.05 \mathrm{M}$ carbonate buffer ( $\mathrm{pH} 9.6$ ). Following stirring at $25^{\circ} \mathrm{C}$ for $6 \mathrm{~h}$, the mixture was dialyzed six times against PBS. The dialysate of the ICA-BSA conjugate was stored at $4^{\circ} \mathrm{C}$ for detection and immunization. The ICA-OVA conjugate was synthesized using the same method described above.

Animal treatment. A total of 5 female BALB/c mice (6 weeks old) were purchased from Vital River Laboratories (Beijing, China). The mice were fed a standard rodent diet (Keaoxieli Animal Feed Co., Ltd., Beijing, China) ad libitum and housed in an environmentally controlled $\left(23 \pm 2^{\circ} \mathrm{C} ; 12 \mathrm{~h}\right.$ light/dark cycle) animal facility. Mice were sacrificed by cervical dislocation. The present study was performed according to the Guidelines for the Care and Use of Laboratory Animals and was approved by the Joint Ethical Review Committee of the Beijing University of Chinese Medicine (Beijing, China; approval no. 2013 BZHYLL00106).

Immunization. The immunizations were performed at 2-week intervals. The mice were subcutaneously injected with a $50 \mu \mathrm{g}$ volume of the ICA-BSA conjugate in PBS, emulsified with an equal volume of Freund's complete adjuvant in the initial immunization. The second and third immunizations, which contained $50 \mu \mathrm{g}$ of the ICA-BSA conjugate in Freund's incomplete adjuvant, were injected subcutaneously 2 and 4 weeks following the initial injection. Blood was obtained from the tail vein of the mice and centrifuged at $4^{\circ} \mathrm{C}$ and $2,227 \times g$ for $10 \mathrm{~min}$ following the third immunization, and a titre of sera was examined by indirect ELISA, using ICA-OVA as the solid-phase antigen. After 2 weeks the fourth immunization involved injection with a solution of ICA-BSA $(100 \mu \mathrm{g})$ in PBS without adjuvant.

Cell fusion and preparation of the anti-ICA MAb. At 3 days following the final immunization the spleen from immunized mice was removed using a cell strainer. Using the head of a clean syringe, the spleen was ground to dissociate the splenocytes. The cells were suspended following filtering 
and splenocytes were isolated and fused with the hypoxanthine-aminopterin-thymidine (HAT)-sensitive SP2/0 mouse myeloma cell line (ScienCell Research Laboratory; Carlsbad, CA, USA), according to the PEG method $(24,25)$. Briefly, following centrifugation at room temperature at $180 \times \mathrm{g}$ for $10 \mathrm{~min}$ of the blended splenocytes and myeloma cells (at a ratio of 5:1), $1 \mathrm{ml}$ of PEG was added drop-wise to the cell pellet and then incubated for $1 \mathrm{~min}$ at $37^{\circ} \mathrm{C}$. The HAT medium (Sigma-Aldrich; Merck Millipore) was then added. The hybridoma was transferred to 96-well plates for cell culture. The cells producing MAbs reactive to ICA, as identified using indirect ELISA, were cloned according to the limiting dilution method (26). The established hybridoma was then cultured in the $\mathrm{HT}$ medium with $5 \% \mathrm{CO}_{2}$ at $37^{\circ} \mathrm{C}$ for 10 days.

A total of 20 mice (10-week-old, male) were purchased from Vital River Laboratories (Beijing, China). The mice were fed a standard rodent diet (Keaoxieli Animal Feed Co., Ltd., Beijing, China) ad libitum and housed in an environmentally controlled $\left(23 \pm 2^{\circ} \mathrm{C} ; 12 \mathrm{~h}\right.$ light/dark cycle) animal facility, which had been injected with $300 \mu$ l Freund's incomplete adjuvant on the previous day. The hybridomas were transplanted into the abdominal cavity of 10 -week-old male BALB/c mice, which had been injected with Freund's incomplete adjuvant on the previous day. The mice were sacrificed prior to the injection by cervical dislocation. After 5-7 days, fluid was drained from the resulting ascites. This fluid was then purified by centrifugation at $4^{\circ} \mathrm{C}$ and $5,702 \times \mathrm{g}$ for $10 \mathrm{~min}$, followed by caprylic acid precipitation and protein quantification (27).

Establishment of the icELISA method. The reactivity of the anti-ICA MAbs with ICA-OVA was determined using indirect ELISA (iELISA). The ICA-OVA was dissolved in CBS, following which $100 \mu \mathrm{l}$ was added to each well of a 96-well maxisorp immunoplate and incubated for $1 \mathrm{~h}$. Each well was then treated with $200 \mu \mathrm{l}$ of GPBS for $1 \mathrm{~h}$ to inhibit non-specific absorption. The plate was washed three times with PBST prior to the addition of $100 \mu \mathrm{l}$ of anti-ICA MAbs $(1: 10,0000)$. Following incubation for $1 \mathrm{~h}$, the plate was washed three times and incubated with $100 \mu$ of peroxidase-labelled goat anti-mouse IgG solution (1:10,000; cat. no. C1308; Applygen Technologies Inc., Beijing, China) for $30 \mathrm{~min}$. The plate was then washed three times, and $100 \mu \mathrm{l}$ of TMB substrate solution was added to each well, followed by incubation for $15 \mathrm{~min}$. The reaction was terminated by adding $50 \mu \mathrm{l}$ of $2 \mathrm{M} \mathrm{H}_{2} \mathrm{SO}_{4}$, and the absorbance was measured at $450 \mathrm{~nm}$ using a BioTek ELx 800 microplate reader (BioTek China, Beijing Chin). All reactions were performed at $37^{\circ} \mathrm{C}$.

The reactivity of the anti-ICA MAbs was determined using an icELISA. The protocol for this assay was identical to that used for the iELISA, with the exception that the primary antibodies used were $50 \mu \mathrm{l}$ of ICA and $50 \mu \mathrm{l}$ of anti-ICA MAb, and the subsequent incubation duration was $1 \mathrm{~h}$.

Assay sensitivity and specificity. The icELISA was established with ICA-OVA $(1: 2,000)$ as the solid-phase antigen and ascites induced by the transplant of the hybridoma $(1: 200,000)$. Various quantities of ICA were added to compete with the coated antigen, by which the standard curve of inhibition and measuring range were established.
Cross-reactivity (CR) is the most important factor in phytochemical investigations, as there are several structurally-related compounds. In the present study, the assay specificities were examined using icELISA with various associated compounds; the CR\% of ICA and the associated compounds were determined according to Weiler's equation (28).

Assay variation. Intra-assay variation was assessed by evaluating the relative standard deviation (RSD\%) of the ICA samples of varying concentrations $(20,40,160$ and $640 \mathrm{ng} / \mathrm{ml})$ plated in six replicates across a microtitre plate. Inter-assay variation was determined by evaluating the ICA samples on three different microtitre plates for 3 days consecutively.

Recovery. The ICA stock solution was spiked into PBS solutions at different volumes (0, 100, 200,400 and $800 \mathrm{ng} / \mathrm{ml})$, and recovery was determined using icELISA, as described above. The ratio of the value obtained for the known concentration was used to evaluate the matrix effect.

Quantitative analysis of ICA using HPLC. HPLC analysis was performed according to a previously reported protocol (29) with modifications. The HPLC system used in the present study was an Agilent 1260 Infinity with an Agilent ZORBAX SB-C18 column (Agilent Technologies, Inc., Santa Clara, CA, USA; $5 \mu \mathrm{m} ; 0.46 \times 150 \mathrm{~mm}$ ), maintained at room temperature. The components were separated by gradient elution using water (solvent A) and acetonitrile (solvent B) at a constant flow rate of $1.0 \mathrm{ml} \mathrm{min}$. The isocratic profile was as follows: $30 \%$ solvent B for 0-20 min. Each sample $(10 \mathrm{ml})$ was injected and monitored at $270 \mathrm{~nm}$. The column was held at $30^{\circ} \mathrm{C}$.

Sample preparation. Dried samples were pulverized and extracted with $50 \mathrm{ml}$ of $70 \%$ aqueous ethanol solution in an ultrasonic bath at $50^{\circ} \mathrm{C}$ for $30 \mathrm{~min}$ prior to filtering. The processed samples were analyzed using HPLC. The samples were diluted in the effective measuring range of 10 to $1,000 \mathrm{ng} / \mathrm{ml}$ analyzed using icELISA.

Correlation between HPLC and ELISA for the analyses of total ICA in TCM prescriptions using MAb. In the present study, four types of TCM prescription were prepared in accordance with the traditional method (15). The Folium Epimedii extract and other herb extracts were prepared, as follows: Powdered herbs $(30 \mathrm{~g})$ were extracted with boiling water $(60 \mathrm{ml})$ for $30 \mathrm{~min}$ and filtered through gauze. Following the addition of ethanol to $70 \%$, the solution was stored at $4^{\circ} \mathrm{C}$ overnight and filtered. Subsequently, the solvents were vaporized to obtain dry powders, which were diluted with distilled water $(10 \mathrm{ml})$. The samples were diluted in the effective measuring range of 10 to $1,000 \mathrm{ng} / \mathrm{ml}$ analyzed using icELISA.

\section{Results and Discussion}

Production of MAb against ICA. Six-week-old female BALB/c mice were immunized with the ICA-BSA conjugate, and the desired hybridoma-secreting MAb against ICA was cloned using the limiting dilution method following screening using icELISA. The anti-ICA MAb was successfully obtained for further experiments. 
Table I. Cross-reactivities of anti-icariin monoclonal antibodies against natural occurring compounds.

\begin{tabular}{lc}
\hline Compound & Cross-reactivity (\%) \\
\hline Polygala acid & 0.20 \\
Quercetin & 0.26 \\
Puerarin & $<0.09$ \\
Baicalin & $<0.09$ \\
Daidzin & $<0.09$ \\
Scutellarein & $<0.09$ \\
Hesperidin & $<0.09$ \\
Naringin & $<0.09$ \\
Quercetin & $<0.09$ \\
Hyperoside & $<0.09$ \\
Geniposide & $<0.09$ \\
Vitexin & $<0.09$ \\
Curculigoside & $<0.09$ \\
Rutin & $<0.09$ \\
Paeoniflorin & $<0.09$ \\
Ginsenoside Rg1 & $<0.09$ \\
Saikosaponin A & $<0.09$ \\
\hline
\end{tabular}

Table II. Intra- and inter-assay precisions of ICA analysis via an enzyme-linked immunosorbent assay using anti-ICA monoclonal antibody.

\begin{tabular}{lcc}
\hline & \multicolumn{2}{c}{ Relative standard deviation $(\%)$} \\
\cline { 2 - 3 } ICA $(\mathrm{ng} / \mathrm{ml})$ & Intra-assay & Inter-assay \\
\hline 20 & 3.76 & 6.50 \\
40 & 2.51 & 7.13 \\
160 & 3.44 & 9.84 \\
640 & 2.33 & 5.56 \\
\hline
\end{tabular}

Values presented reflect the mean standard deviation of three plates with six replicate wells for each concentration within one plate from 3 days consecutively. ICA, icariin.

Assay sensitivity and specificity. As shown in Fig. 1, competitive inhibition occurred between the MAb and ICA-OVA with various concentrations of ICA, resulting in a calibration curve for ICA, analyzed using icELISA. The concentrations of the standard solutions were 9.77, 19.53, 39.06, 78.12, 156.25, $312.50,625.00$ and 1,250.00 $\mathrm{ng} / \mathrm{ml}$. Under these conditions, a linear regression coefficient of 0.9828 and linear regression equation, $\mathrm{y}=-0.119 \operatorname{Lg}(\mathrm{x})+1.3644(9.77 \mathrm{ng} / \mathrm{ml}-1.25 \mu \mathrm{g} / \mathrm{ml})$, were achieved with a half maximal inhibitory concentration of $156 \mathrm{ng} / \mathrm{ml}$. The full measuring range of the assay extended between 10 and $1,000 \mathrm{ng} / \mathrm{ml}$.

As shown in Table I, several compounds associated with ICA were found to have cross-reactivity with the anti-ICA MAb, including polygala acid $(0.20 \%)$ and quercetin $(0.26 \%)$. The MAb had no cross-reactivity with other compounds $(<0.09 \%)$.
Table III. Recoveries of ICA from samples with various concentrations of ICA added, determined by enzyme-linked immunosorbent assay using anti-icariin monoclonal antibody.

\begin{tabular}{ccc}
\hline $\begin{array}{l}\text { ICA added } \\
(\mathrm{ng} / \mathrm{ml})\end{array}$ & $\begin{array}{c}\text { Quantity } \\
\text { measured (ng/ml) }\end{array}$ & Recovery (\%) \\
\hline 0 & $42.24 \pm 4.54$ & \\
100 & $133.42 \pm 13.44$ & 93.80 \\
200 & $256.88 \pm 88.69$ & 106.15 \\
400 & $525.10 \pm 49.70$ & 118.80 \\
800 & $852.66 \pm 27.16$ & 101.27 \\
\hline
\end{tabular}

Data for the quantity measured are presented as the mean \pm standard deviation and recovery percentages are presented as the mean values from triplicate samples. $0 \mathrm{ng} / \mathrm{ml}$ ICA was used as a control. Recovery was calculated as follows: Recovery $(\%)=$ (quantity measured - control) / quantity added x 100\%. ICA, icariin.

Table IV. Contents of ICA in four traditional Chinese medicines using ELISA with anti-ICA monoclonal antibodies and HPLC.

\begin{tabular}{lccccc}
\hline & \multicolumn{2}{c}{ ELISA } & & \multicolumn{2}{c}{ HPLC } \\
\cline { 2 - 3 } \cline { 5 - 6 } Sample & ICA $(\mu \mathrm{g} / \mathrm{ml})$ & CV $(\%)$ & & ICA $(\mu \mathrm{g} / \mathrm{ml})$ & CV $(\%)$ \\
\hline 1 & $27.61 \pm 6.46$ & 7.17 & & $30.62 \pm 0.37$ & 1.20 \\
2 & $253.94 \pm 9.78$ & 3.85 & & $230.69 \pm 1.60$ & 0.70 \\
3 & $105.68 \pm 8.07$ & 7.64 & & $103.62 \pm 1.19$ & 1.15 \\
4 & $312.79 \pm 18.57$ & 5.94 & & $398.57 \pm 0.37$ & 0.22 \\
\hline
\end{tabular}

Data are presented as the mean \pm standard deviation from six wells for each sample. ICA, icariin; ELISA, enzyme-linked immunosorbent assay; HPLC, high-performance liquid chromatography; $\mathrm{CV}$, coefficient of variation.

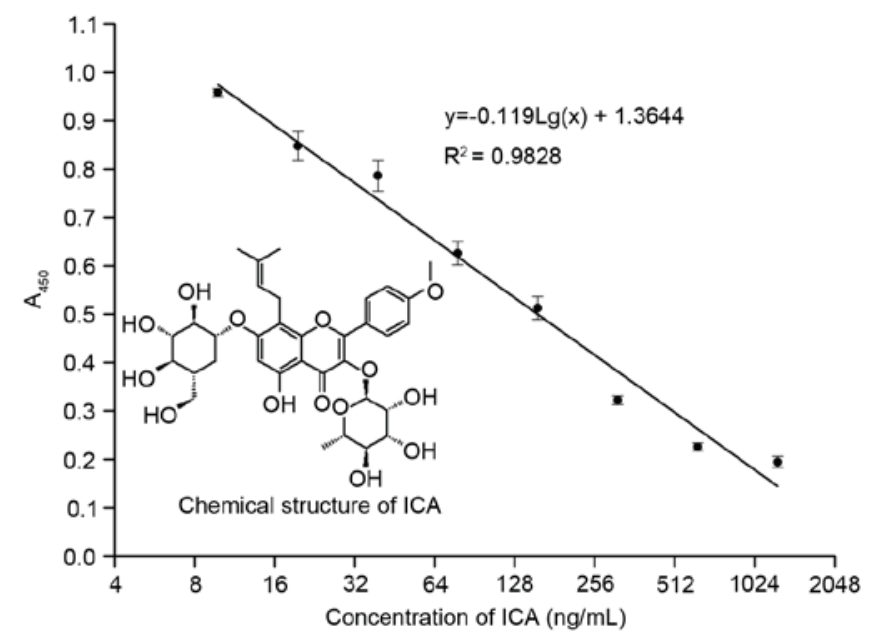

Figure 1. Calibration curve of icariin determined using an indirect competitive enzyme-linked immunosorbent assay with monoclonal antibody against ICA. Data are presented as the mean \pm standard deviation $(n=3)$. ICA, icariin; $\mathrm{A}_{450}$, absorbance in the presence of ICA. 
Assay variation. Reproducibility and precision are important criteria for an immunoassay. Standard curves for the ICA analyzed using icELISA on 3 days (consecutive) were compared and the variations were calculated. The variations between the well-to-well (intra-assay) and plate-to-plate (inter-assay) replicates were measured. As shown in Table II, the intra-assay RSDs were $<4 \%$ and the inter-assay RSDs were $<10 \%$, which indicated that this assay was accurate and stable.

Recovery of ICA by icELISA with spiked-in samples. Various quantities of ICA were added to the Folium Epimedii extract, following which the spiked-in samples were dissolved and mixed evenly, and ICA content was calculated using icELISA. For each level, six samples were analyzed. The levels of ICA in the Folium Epimedii extract were determined using icELISA. ICA recovery ranged between 93.80 and $118.80 \%$, with an average of $105.00 \%$ (Table III).

Quantitative analysis of ICA in various TCM prescriptions using icELISA. In the present study, the four TCM prescriptions, which varied in their composition ratios, were determined using icELISA without pre-treatment. The concentrations of ICA in the four TCM prescriptions are shown in Table IV.

Correlation between HPLC and ELISA analyses of total content of ICA using MAb. Powdered samples were extracted as described above. As shown in Table IV, the contents determined using ELISA were consistent with those determined using HPLC.

The results of the experiments in the present study showed that the anti-ICA MAb had high sensitivity and specificity, and that the icELISA method had its own unique advantages, compared with HPLC. It is typical that intra-assay variations are generally lower, compared with inter-assay variations. The factors contributing to these variations are considered to include the hapten quality, coating, plate wells and multichannel pipettor, edge effects due to evaporation, uneven temperature during incubation, and day-to-day variation in the preparation of the reagents. Thus, the creation of a novel standard curve is required every time to reduce the variation.

To the best of our knowledge, the present study developed the first MAb against ICA and established the subsequent ELISA method, which provided a simpler, more efficient and sensitive approach for determining the ICA content in drug materials and biological samples. This method can also serve as a useful tool for investigating the pharmacokinetics and targets of ICA.

\section{Acknowledgements}

The present study was supported by the National Natural Science Foundation of China (grant nos. 81473338 and 81373542), the National Key Basic Research Development Program (973 program; grant no. 2011CB505101) and the Classical Prescription Basic Research Team of Beijing University of Chinese Medicine. The authors would like to thank Elsevier Language Editing Services for providing language assistance.

\section{References}

1. Zhang D, Yuan C, Zhu Z, Jin X and Li L: Influence of the mixture of Epimedii Herba and ginkgo folium extracts on the coronary flow of isolated hearts in rats. Pharmacogn Mag 9: 290-293, 2013

2. Zhou H, Yuan Y, Liu Y, Deng W, Zong J, Bian ZY, Dai J and Tang QZ: Icariin attenuates angiotensin II-induced hypertrophy and apoptosis in H9c2 cardiomyocytes by inhibiting reactive oxygen species-dependent JNK and p38 pathways. Exp Ther Med 7: 1116-1122, 2014.

3. Zhang DW, Cheng Y, Wang NL, Zhang JC, Yang MS and Yao XS: Effects of total flavonoids and flavonol glycosides from Epimedium koreanum Nakai on the proliferation and differentiation of primary osteoblasts. Phytomedicine 15: 55-61, 2008.

4. Wang Q, Hao J, Pu J, Zhao L, Lü Z, Hu J, Yu Q, Wang Y, Xie Y and Li G: Icariin induces apoptosis in mouse MLTC-10 Leydig tumor cells through activation of the mitochondrial pathway and down-regulation of the expression of piwil4. Int J Oncol 39: 973-980, 2011.

5. Xu CQ, Liu BJ, Wu JF, Xu YC, Duan XH, Cao YX and Dong JC: Icariin attenuates LPS-induced acute inflammatory responses: Involvement of PI3K/A kt and NF-kappaB signaling pathway. Eur J Pharmacol 642: 146-153, 2010.

6. Song YH, Cai H, Gu N, Qian CF, Cao SP and Zhao ZM: Icariin attenuates cardiac remodelling through down-regulating myocardial apoptosis and matrix metalloproteinase activity in rats with congestive heart failure. J Pharm Pharmacol 63: 541-549, 2011.

7. Tong JS, Zhang QH, Huang X, Fu XQ, Qi ST, Wang YP, Hou Y, Sheng J and Sun QY: Icaritin causes sustained ERK1/2 activation and induces apoptosis in human endometrial cancer cells. PLoS One 6: e16781, 2011.

8. Guo Y, Zhang X, Meng J and Wang ZY: An anticancer agent icaritin induces sustained activation of the extracellular signal-regulated kinase (ERK) pathway and inhibits growth of breast cancer cells. Eur J Pharmacol 658: 114-122, 2011

9. Sun P, Liu Y, Deng X, Yu C, Dai N, Yuan X, Chen L, Yu S, Si W, Wang X, et al: An inhibitor of cathepsin K, icariin suppresses cartilage and bone degradation in mice of collagen-induced arthritis. Phytomedicine 20: 975-979, 2013.

10. Li ZJ, Yao C, Liu SF, Chen L, Xi YM, Zhang W and Zhang GS: Cytotoxic effect of icaritin and its mechanisms in inducing apoptosis in human burkitt lymphoma cell line. Biomed Res Int 2014: 391512, 2014.

11. Jin J, Li Y, Kipletting Tanui E, Han L, Jia Y, Zhang L, Wang Y, Zhang $X$ and Zhang Y: Fishing and knockout of bioactive compounds using a combination of high-speed counter-current chromatography (HSCCC) and preparative HPLC for evaluating the holistic efficacy and interaction of the components of Herba Epimedii. J Ethnopharmacol 147: 357-365, 2013.

12. Pozharitskaya ON, Kosman VM, Shikov AN, Demchenko DV, Eschenko AY and Makarov VG: Comparison between HPLC and HPTLC densitometry for the determination of icariin from Epimedium koreanum extracts. J Sep Sci 30: 708-712, 2007.

13. Xu T, Wei KY, Wang J, Eremin SA, Liu SZ, Li QX and Li J: Development of an enzyme-linked immunosorbent assay specific to Sudan red I. Anal Biochem 405: 41-49, 2010.

14. Daduang S, Sattayasai N, Sattayasai J, Tophrom P, Thammathaworn A, Chaveerach A and Konkchaiyaphum M: Screening of plants containing Naja naja siamensis cobra venom inhibitory activity using modified ELISA technique. Anal Biochem 341: 316-325, 2005.

15. Zhao Y, Kong H, Sun Y, Feng H, Zhang Y, Su X, Qu H and Wang Q: Assessment of baicalin in mouse blood by monoclonal antibody-based icELISA. Biomed Chromatogr 28: 1864-1868, 2014.

16. Qu H, Zhang G, Li Y, Sun H, Sun Y, Zhao Y and Wang Q: Development of an enzyme-linked immunosorbent assay based on anti-puerarin monoclonal antibody and its applications. J Chromatogr B Analyt Technol Biomed Life Sci 953-954: 120-125, 2014.

17. Qu HH, Sun Y, Wu TT, Zhang GL, Cheng JJ, Wang XQ, Feng HB, Zhao Y and Wang QG: Pharmacokinetics of geniposide by monoclonal antibody-based icELISA in mice after oral administration of Huanglian-Jiedu-Tang. Biol Pharm Bull 37: 1525-1533, 2014. 
18. Zhang Y, Qu H, Zeng W, Zhao Y, Shan W, Wang X, Wang Q and Zhao Y: Development of an enzyme-linked immunosorbent assay and immunoaffinity chromatography for glycyrrhizic acid using an anti-glycyrrhizic acid monoclonal antibody. J Sep Sci 38: 2363-2370, 2015.

19. Zhao Y, Qu H, Wang X, Zhang Y, Shan W, Zhao Y and Wang Q: A sensitive and specific indirect competitive Enzyme-linked immunosorbent assay for detection of paeoniflorin and its application in pharmacokinetic interactions between paeoniflorin and glycyrrhizinic acid. Planta Med 81: 765-770, 2015.

20. Qu H, Sai J, Wang Y, Sun Y, Zhang Y, Li Y, Zhao Y and Wang Q Establishment of an enzyme-linked immunosorbent assay and application on determination of ginsenoside Re in human saliva. Planta Med 80: 1143-1150, 2014.

21. Qu H, Wang Y, Shan W, Zhang Y, Feng H, Sai J, Wang Q and Zhao Y: Development of ELISA for detection of Rh1 and Rg2 and potential method of immunoaffinity chromatography for separation of epimers. J Chromatogr B Analyt Technol Biomed Life Sci 985: 197-205, 2015.

22. Phrompittayarat W, Putalun W, Tanaka H, Jetiyanon K, Wittaya-Areekul S and Ingkaninan K: Determination of pseudojujubogenin glycosides from Brahmi based on immunoassay using a monoclonal antibody against bacopaside I. Phytochem Anal 18: 411-418, 2007.
23. Zhu S, Shimokawa S, Shoyama Y and Tanaka H: A novel analytical ELISA-based methodology for pharmacologically active saikosaponins. Fitoterapia 77: 100-108, 2006.

24. Galfrè G and Milstein C: Preparation of monoclonal antibodies: Strategies and procedures. Methods Enzymol 73 . 3-46, 1981.

25. North SM, Styles JM, Hobbs SM and Dean CJ: Monoclonal antibodies to rat sarcomata. I. Immunization procedures and source of lymphoid cells for hybridoma production. Immunology 47: 397-405, 1982.

26. Goding JW: Antibody production by hybridomas. J Immunol Methods 39: 285-308, 1980.

27. Liu HF, Ma J, Winter C and Bayer R: Recovery and purification process development for monoclonal antibody production. MAbs 2: 480-499, 2010.

28. Auten AA, Beauchamp LN, Taylor J and Hardinger KL: Hidden sources of grapefruit in beverages: Potential interactions with immunosuppressant medications. Hosp Pharm 48: 489-493, 2013.

29. Jian Z, Xu G, Chen H, Wang $H$ and Hu X: Study on the differences of major pharmaceutical ingredients in different parts and processed medicinal material of Epimedium brevicornu maxim in Taihang mountain. Nutr Hosp 32: 913-917, 2015. 